\title{
Efectividad de la anestesia intraarticular en artroscopía de rodilla
}

\author{
Effectiveness of intra-articular anesthesia in knee arthroscopy \\ Martínez-Navarro JL, * Espinoza-Castolo M, ${ }^{\ddagger}$ Vargas-Vargas $\mathrm{SF}^{\S}$ \\ UMAE No. 1, IMSS. Bajío, Zapopan, Jalisco, México.
}

RESUMEN. Introducción: La artroscopía de rodilla es uno de los procedimientos que más comúnmente se realizan para tratar esta articulación. La anestesia general y el bloqueo espinal son las modalidades de anestesia más comunes para este procedimiento. Consideramos que esta cirugía se puede manejar con anestesia local intraarticular, con lo que se evitan estancias en recuperación más prolongadas; tiene un mejor manejo efectivo del dolor y optimización de tiempos prequirúrgicos, intraquirúrgicos y postquirúrgicos. Material y métodos: Estudio prospectivo, cuasiexperimental y longitudinal, realizado para comparar dos procedimientos anestésicos: la anestesia intraarticular (lidocaína con epinefrina, bupivacaína y fentanil) sin uso de isquemia (grupo A) y el bloqueo espinal y uso de manguito de isquemia (grupo B). Ambos procedimientos se calcularon mediante la evaluación de la edad, el tiempo de cirugía, el dolor transquirúrgico y a las 24 horas mediante escala EVA, el tiempo de estancia en recuperación, la necesidad de administrar anestesia general o dosis anestésica de rescate y los grados de flexión a las 24 horas en el postquirúrgico. Se utilizó prueba t de Student con un nivel de significación $\mathrm{p}<0.05$ para variables no paramétricas y $\chi^{2}$ para variables paramétricas. Resultados: Se incluyeron 38 pacientes por cada grupo. El tiempo quirúrgico fue de 45 contra 39 minutos $(\mathrm{p}=0.03)$; el tiempo en recuperación: 1.9 contra 3 horas $(p=0.0004)$, el cual fue menor en el grupo $\mathrm{A}$, mientras que el dolor transquirúrgico fue mayor (17 contra $3 \%[p=0.09])$, sin requerir anestesia general. Conclusiones: Las ventajas a favor de la anestesia intraarticular fueron: estadías hospitalarias cortas, menor dolor a las 24 horas y seguridad en su aplicación. Su desventaja es la probabilidad de dolor transquirúrgico, siendo suficiente la sedación.

Palabras clave: Artroscopía, rodilla, anestesia, local, regional, cirugía.
ABSTRACT. Indroduction: Knee arthroscopy is one of the most commonly performed procedures. General anesthesia and spinal blockage are the most common forms of anesthesia. We consider that this surgery can be managed with intra-articular local anesthesia, thus avoiding prolonged stays in recovery, with effective pain management and optimization of presurgical, intrasurgical and post-surgical times. Material and methods: Prospective, quasi-experimental, longitudinal study to compare two anesthetic procedures: intraarticular anesthesia (lidocaine with epinephrine, bupivacaine and fentanyl) without the use of ischemia (group A); spinal blockage and use of ischemia (group B) by assessing age, surgery time, pain during surgery and 24 hour post-op VA scale, time spent in recovery, need to administer general anesthesia or anesthetic rescue doses, 24-hour range of motion. Student t-test, significance level was used: $p$ $<0.05$ for nonparametric variables and $\chi^{2}$ parametric variables. Results: 38 patients were included per group. The surgical time (45 min vs $39 \mathrm{~min}, \mathrm{p}=0.03$ ), recovery time ( 1.9 vs 3 hours, $p=0.0004)$ were lower in group A, while pain during surgery was greater ( 17 vs $3 \%, p=$ 0.09), without requiring general anesthesia. Conclusion: The advantages in favor of intraarticular anesthesia were: shortened hospital stays, less pain at 24 hours, safety in its application. Its disadvantage is the possibility of pain during surgery and sedation is sufficient as adjuvant.

Keywords: Arthroscopy, knee, anesthesia, local, regional, surgery.

\section{Nivel de evidencia: IV. Series de casos.}

* Residente del cuarto año de Ortopedia y Traumatología.

* Médico adscrito a Ortopedia y Traumatología, Módulo de rodilla.

${ }^{\S}$ Médico adscrito a Ortopedia y Traumatología, Módulo de artroscopía.

Dirección para correspondencia:

Dr. Jorge Luis Martínez Navarro

Unidad Médica de Alta Especialidad (UMAE) Núm. 1 Bajío.

Isaac Newton Núm. 3983, Col. Arboledas, Zapopan, C.P. 45070, Jalisco, México.

E-mail: jolmanx@gmail.com 


\section{Introducción}

La artroscopía de rodilla es, en la actualidad, una de las cirugías electivas más comúnmente realizadas en ortopedia. Algunas indicaciones para su realización son por ruptura meniscal, síntomas mecánicos, quistes meniscales, lesiones condrales, condromalacia, plica sintomática y enfermedad articular degenerativa. Esta cirugía es de mínima invasión, cuyo tratamiento postquirúrgico inmediato puede llevarse a cabo de manera ambulatoria. En la actualidad, en nuestro centro hospitalario contamos con experiencia al usar bloqueo neuroaxial mediante el internamiento y hospitalización de, al menos, 24 horas.

El uso de anestésicos locales con una mezcla de lidocaína y bupivacaína, tanto de los sitios de los portales como intraarticulares, ha demostrado ser exitoso en artroscopías básicas; cualquier manipulación ósea, ${ }^{1,2}$ al igual que el uso de torniquete tendrían que ser minimizados. ${ }^{1}$ Este uso local ha demostrado seguridad sin incrementar los efectos secundarios de la ropivacaína en única dosis intraarticular, ${ }^{3}$ así como la seguridad del uso de bupivacaína sola y bupivacaína más morfina. ${ }^{4}$

La anestesia regional con sedación o anestesia espinal son opciones apropiadas para procedimientos artroscópicos, especialmente cuando existe la presencia de comorbilidades que limiten la anestesia general; como ventaja, se disminuiría las admisiones hospitalarias en caso de eventos quirúrgicos que involucren la manipulación de hueso. Entre las desventajas están la necesidad de inmovilizar la extremidad por lo menos hasta recuperar la sensibilidad de manera completa y la hospitalización obligatoria. ${ }^{1,5,6}$

Respecto a la infiltración intraarticular con $20 \mathrm{ml}$ al $0.25 \%$ de bupivacaína, tiene como resultado la disminución en hasta 20 horas postquirúrgicas. Ahora bien, el uso postquirúrgico de $10 \mathrm{ml}$ al $0.5 \%$ de bupivacaína mejora el dolor evaluado con la Evaluación Visual Análoga (EVA). ${ }^{7}$

En el año 2000, en la Revista Mexicana de Ortopedia y Traumatología se publicó un ensayo clínico controlado y doble ciego con dos grupos de 15 pacientes. En el primer grupo se administró intraarticularmente bupivacaína al $0.5 \%$ con epinefrina 1: $200,000+100 \mu \mathrm{g}$ de fentanilo en presentación de $30 \mu \mathrm{g}$; en el segundo grupo se administró intraarticularmente bupivacaína al $0.5 \%$ con epinefrina 1:200,000. El objetivo fue comparar el efecto anestésico transoperatorio y el efecto analgésico postoperatorio entre ambos grupos en pacientes mayores de 15 años; el riesgo quirúrgico fue evaluado mediante la escala de la American Society of Anesthesiologists (ASA) I y II con lesión factible de solucionarse por artroscopía. No hubo diferencia significativa del resultado en la EVA transoperatoria $(p=0.4932)$, pero sí hubo una diferencia significativa en la EVA postoperatoria $(\mathrm{p}=0.0353)$. El estudio concluyó que la asociación de la bupivacaína y fentanilo ofrece ventajas en el postoperatorio inmediato; en este estudio no se utilizó isquemia. ${ }^{5}$

Otro estudio, realizado por Mondino en 2016 y publicado en la Revista de la Asociación Argentina de Ortopedia y Traumatología, investigó el uso de anestesia raquídea en comparación con la anestesia intraarticular en cirugía artroscópica de rodilla. Dicho trabajo realizó un estudio prospectivo aleatorizado para comparar los procedimientos anestésicos mediante la evaluación de la edad, el tiempo de cirugía, el tiempo de internación, el dolor, la conformidad con el procedimiento y los costos. De esta manera, este análisis describió la presencia de dolor en $28 \%$ de los pacientes en los que se utilizó anestesia intraarticular; en tanto que en aquéllos con bloqueo espinal, sólo 2.8\% presentó dolor. El período de hospitalización fue de 7.34 horas en comparación con el de anestesia intraarticular, que fue de 3.34 horas. La variable costo tuvo una diferencia significativa con respecto a la anestesia raquídea ( $\mathrm{p}<0.0001)$. Aunque los pacientes cursaron con dolor $(\mathrm{p}=0.069)$, se disminuyó el riesgo quirúrgico y la diferencia de costos fue de casi el doble. Se utilizó isquemia sólo en seis pacientes de aquéllos con anestesia raquídea, pero no se usó en ningún paciente del grupo en el que se utilizó anestesia intraarticular. ${ }^{8}$

Así pues, basado en los datos aportados por estos trabajos, el motivo de nuestra investigación es demostrar la efectividad anestésica intraarticular en el paciente operado de artroscopía diagnóstico-terapéutica de rodilla y compararlo con el paciente al que se le realiza bloqueo espinal; con ello, se busca evidenciar que la anestesia intraarticular es posible en todos los pacientes operados de artroscopía sin manipulación ósea, disminuyendo los tiempos prequirúrgicos y transquirúrgicos, y los riesgos propios de complicaciones asociados con el bloqueo regional, para que, en un futuro, pueda reproducirse este método. Además, consideramos que esta cirugía se puede manejar con anestesia local intraarticular, con lo que se evitan estancias prolongadas de recuperación, con un manejo efectivo del dolor y optimización de tiempos prequirúrgicos, intraquirúrgicos y postquirúrgicos. ${ }^{2}$

\section{Material y métodos}

Estudio comparativo, prospectivo, cuasiexperimental y longitudinal, que abarcó de Abril a Diciembre de 2018. La cohorte comparativa se dividió en dos grupos: el grupo A fue para la anestesia intraarticular y el grupo B para el bloqueo espinal, el cual se realiza de manera rutinaria por el Servicio de Anestesiología.

El evento final de evaluación o desenlace fue la presencia de dolor con EVA durante el transquirúrgico y a las 24 horas, el tiempo de estancia en recuperación, la necesidad de sedación, la presencia de eventos adversos y el factor de análisis o de exposición a la anestesia.

\section{Criterios de inclusión}

- Pacientes operados de artroscopía diagnóstica-terapéutica de rodilla.

- Edad entre los 16 y 65 años.

- Cualquier sexo.

- Pacientes operados en nuestro centro hospitalario que aceptaran participar en el estudio y que firmaran el consentimiento informado. 


\section{Criterios de exclusión}

- Índice de masa corporal igual o mayor a 35.

- Pacientes que no aceptaran participar en el estudio.

- Pacientes con artrofibrosis.

- Proceso séptico de rodilla.

- Hemartrosis previa a cirugía.

- Sinovitis aguda.

- Paciente con contraindicación para bloqueo neuroaxial.

\section{Criterios de eliminación}

- Pacientes que no acudieran a consulta en el postquirúrgico.

- Pacientes que se les diagnosticara lesión de ligamento cruzado anterior en el momento de cirugía y se tuviera que realizar reconstrucción.

Muestreo no probabilístico por disponibilidad de casos

De acuerdo con lo reportado por Mondino, ${ }^{5}$ quien menciona que $28 \%$ de los sujetos sometidos a anestesia intraarticular presentó dolor comparado con $5.7 \%$ de los sujetos sometidos a anestesia espinal $y$, asumiendo un nivel de significancia del 0.05 , un poder de la prueba de $80 \%$ y considerando $20 \%$ de posibles pérdidas de seguimiento o no respondedores, se obtuvo un tamaño muestral de 38 pacientes por grupo. Se utilizó para ello el programa MedCalc versión 19.1, con la fórmula descrita por Neely.

\section{Manejo estadístico de la información:}

análisis exploratorio

Mediante los valores de sesgo y curtosis con la prueba de Kolmogórov-Smirnov con la corrección de Lilliefors, se identificó si las variables cuantitativas tienen una distribución normal. La estadística descriptiva y las variables cualitativas, como género, presencia de eventos adversos y manejo postquirúrgico, se expresaron como frecuencia y proporciones; las cuantitativas, como edad e intensidad del dolor, mediante promedio y desviación estándar o su equivalente no paramétrico, mediana y percentiles; la estadística inferencial y la comparación de los grupos de las variables cualitativas se realizaron mediante $\chi^{2} 0$ prueba exacta de Fischer, según procediera; las cuantitativas, mediante t de Student o pruebas de Wilcoxon, según procediera. Debido a que se evaluó la presencia del dolor transoperatorio y a las 24 horas por paciente y por grupo, se utilizó ANOVA o su alternativa no paramétrica: Kruskal-Wallis. Se consideró como significativo los valores de p menores a 5\%.

En la consulta preoperatoria se le explicó al paciente el objetivo del estudio, en qué consistía cada una de las dos opciones $\mathrm{y}$, al aceptarlas, firmó el consentimiento informado para ingresar a dicho estudio; posteriormente, se le indicó en qué grupo quedó incluido mediante una aleatorización simple con sobres cerrados.

En el grupo A se utilizó infiltración intraarticular con aguja hipodérmica núm. 22 con bupivacaína isobárica al 5\% a $10 \mathrm{~cm}^{3}$, fentanilo a $4 \mathrm{~cm}^{3}(200 \mu \mathrm{g})$ y lidocaína al $2 \%$ con epinefrina 1:100,000 a $6 \mathrm{~cm}^{3}$ durante un período de latencia de 12 minutos. Los portales de artroscopía se infiltraron con lidocaína al $2 \%$ con epinefrina $1: 100,000$ a $5 \mathrm{~cm}^{3}$ cada uno. ${ }^{2}$ Se administró un antibiótico profiláctico intravenoso a dosis única y analgesia transquirúrgica con $60 \mathrm{mg}$ de ketorolaco intravenoso y $1 \mathrm{~g}$ de metamizol intravenoso. La sedación transanestésica se realizó con 1.5 a $3 \mathrm{mg}$ de midazolam y 50 a $150 \mu \mathrm{g}$ de fentanilo; el apoyo con oxígeno en puntas nasales y monitorización tipo I. A los pacientes se les recetó 500 mg de paracetamol vía oral cada ocho horas y antibiótico profiláctico. No se utilizó isquemia. En aquellos pacientes que requirieran colocar isquemia y/o no toleraran la cirugía por dolor, se complementó su proceso anestésico con anestesia general endovenosa con propofol.

En el grupo B se utilizó la técnica de bloqueo mixto; con bloqueo subaracnoideo con aguja Witacree núm. 27 a través de aguja Tuohy núm. 17 peridural con $6 \mathrm{mg}$ de bupivacaína isobárica al $5 \%$ diluido a $3 \mathrm{~cm}^{3}$ de volumen con líquido cefalorraquídeo; la aplicación se realizó en dirección caudal; al retirar aguja Witacree, se colocó el catéter peridural inerte con dirección caudal, el cual se realizó por un anestesiólogo; se le administró antibiótico profiláctico intravenoso a dosis única y analgesia transquirúrgica con $60 \mathrm{mg}$ de ketorolaco intravenoso y $1 \mathrm{~g}$ de metamizol intravenoso; la sedación transanestésica se realizó con 1.5 a 3 mg de midazolam y de 50 a $150 \mu \mathrm{g}$ de fentanilo; el apoyo con oxígeno en puntas nasales y monitorización tipo I. Se dio receta con $500 \mathrm{mg}$ de paracetamol vía oral cada ocho horas y antibiótico profiláctico. Se utilizó isquemia entre $220-250 \mathrm{mmHg}$ en el tercio proximal del muslo. En aquellos pacientes que no toleraran la cirugía por el dolor, se complementó el proceso anestésico con anestesia general endovenosa con propofol. Al terminar la cirugía, el paciente pasó a recuperación, en donde se mantuvo hasta revertir el efecto anestésico de bloqueo motor con la presencia de fuerza $5 / 5$, de acuerdo con la escala de Daniels.

\section{Resultados}

Durante el período de estudio se incluyó un total de 76 pacientes que requirieron la realización de artroscopía diagnóstica-terapéutica sin necesidad de realizar la reconstrucción ligamentaria: 38 para el grupo de anestesia intraarticular (grupo A) y 38 para el bloqueo espinal (grupo B). En el grupo A 29 pacientes (76\%) fueron del sexo masculino y nueve (24\%) del sexo femenino; en el grupo B $20(53 \%)$ fueron del sexo masculino y 18 (47\%) del sexo femenino. En el grupo A, la edad promedio fue de 35.8 años (rango 18-65) con una desviación estándar de 12.21; en el grupo B, la edad promedio fue de 40.8 años (rango 19-65) con una desviación estándar de 11.86. La edad tuvo una distribución normal según la interpretación de la prueba de Kolmogórov-Smirnov corregida por Lilliefors para ambos grupos. En el grupo A fue más común la intervención en la rodilla izquierda (21\%) que en la derecha (17 casos); por el contrario, 
en el grupo B se operó la rodilla derecha más (25 casos) que la izquierda (13 casos).

Se agruparon los tipos de diagnóstico para su captura: sinovitis, síndrome de mala alineación patelofemoral (SMAPF)/plica sinovial, meniscopatía y condromalacia; y su procedimiento subsecuente: sinovectomía, liberación de retináculo/plica, meniscectomía o sutura meniscal y condroplastía/microfracturas. En el grupo A se registraron tres sinovitis (8\%), nueve SMAPF/plicas sinoviales (24\%), 14 meniscopatías (37\%) y 12 condromalacias (32\%). En tanto que en el grupo B se registraron 10 sinovitis (26\%), 10 SMAPF/plicas sinoviales (26\%), 11 meniscopatías (29\%) y siete condromalacias (18\%). Se tomó el registró de la presencia de complicaciones, cuyo total fue de siete (18\%) pacientes, quienes presentaron derrame articular; de éstos, uno fue en el grupo A con el diagnóstico de condromalacia y seis (15\%) se encontraron en el grupo B, siendo tres $(50 \%)$ con el diagnóstico de sinovitis, dos (33\%) con el diagnóstico de plica y un paciente (17\%) con el diagnóstico de condromalacia.

Hubo variación en cuanto al tiempo quirúrgico. En el grupo A fue de 39.3 minutos (rango 25-55); en el grupo B el promedio fue de 45.28 minutos (25-120). Esta diferencia se encontró significativa $(p=0.03)$. En cuanto a la isquemia, no fue necesaria en ningún paciente del grupo A, mientras que siempre se utilizó en el grupo B.

En cuanto al dolor transquirúrgico, siete (17\%) pacientes presentaron dolor durante el procedimiento; de los cuales, dos $(5 \%)$ requirieron dosis de rescate a consideración por el Servicio de Anestesiología; mientras tanto, sólo un paciente presentó dolor transquirúrgico, requiriendo dosis de rescate a consideración por el Servicio de Anestesiología. La EVA promedio en el grupo A fue $0.5 \mathrm{y}$ en el grupo B fue 0.157 . La diferencia sí fue significativa, siendo más dolorosa en el transquirúrgico $(\mathrm{p}=0.015)$ en el grupo A.

La estancia en recuperación en el grupo A tuvo una media de 1.9 horas (rango 1-4, DE 0.7); el grupo B tuvo una media de tres horas (rango 0-5, DE 1.43); esta diferencia también fue significativa $(\mathrm{p}=0.0004)$. Se hospitalizó por dolor a un paciente del grupo A (3\%); en el grupo B se hospitalizaron a cuatro (11\%) pacientes. De los sujetos que requirieron hospitalización en el grupo B, en dos $(50 \%)$ se realizaron microfracturas, en uno (25\%) liberación de retináculo y en uno (25\%) con lesión compleja de menisco; al paciente que requirió hospitalización en el grupo B se realizó microfracturas; ningún paciente requirió anestesia general.

A las 24 horas, el dolor reportado en la EVA fue menor, en promedio, en el grupo A (1.29 rango 1-6) que en el grupo B (1.5, rango 0-4). Esta diferencia no fue significativa (0.09). Se presentó dolor a las 24 horas en EVA 0 en 13 sujetos, EVA 1 en 10, EVA 2 en 7, EVA 3 en 8; ninguno presentó EVA mayor a 4 en el grupo A. En el grupo B, se presentó dolor a las 24 horas en EVA 0 en 16 sujetos, EVA 1 en 1 sujeto, EVA 2 en 12 sujetos, EVA 3 en 2 sujetos y EVA 4 en 8 sujetos.

No hubo complicaciones tras los procedimientos, aunque sí incidentes (derrame articular) en seis pacientes (15\%) del grupo B; sólo un paciente del grupo A (3\%) presentó derra- me articular. Aislando el procedimiento de microfracturas, no fue significativa (prueba $\chi^{2}=0.34$ ) para mayor probabilidad de derrame o de requerir hospitalización frente al resto de diagnósticos (prueba $\chi^{2}=0.5$ ) (Figura 1).

La capacidad de flexión a las 24 horas fue ligeramente mayor en el grupo A con 91 grados como mediana (rango: 50-120; desviación estándar: 13.4); mientras que en el grupo B fue de 85 grados de mediana (rango de 30-110; desviación estándar 22.4). Estas diferencias no fueron significativas $(\mathrm{p}=0.12)$. Estos resultados se resumen en la Tabla 1. Finalmente, no se presentó ningún caso de condrólisis en nuestra muestra.

\section{Discusión}

Se presentó una disminución del tiempo quirúrgico de manera significativa ( 35 contra 40 minutos), cercano a los cinco minutos, ya vista en estudios previos, tal y como lo describió Mondino, ${ }^{5}$ siendo el tiempo con el uso de anestesia intraarticular similar al reportado por Martínez Trejo. ${ }^{8}$ Consideramos la diferencia con Mondino (35 en el grupo de anestesia intraarticular contra 32 minutos), debido a la presencia de residentes, tal y como también Vidal ${ }^{9}$ menciona el apoyo de residentes. La presencia de dolor transquirúrgico $(26 \%)$ en el grupo estudiado es similar a la serie reportada por Martínez Trejo ${ }^{8}(26 \%)$ y menor que en la reportada por Mondino; ${ }^{5}$ esto último debido quizá al uso de lidocaína con epinefrina solamente, similar en ambos estudios (38\% para el grupo B de Martínez Trejo y 33\% para el grupo estudio de Mondino).

El análisis del dolor postquirúrgico a las 24 horas, aunque no es el objetivo principal de este protocolo, es bueno en nuestro estudio, con sólo 21\% alcanzando dolor con EVA mayor a 3; en otros estudios, este patrón no se repite $(85 \%$ con EVA mayor a 3), ${ }^{8}$ aunque se podría asociar con el uso rutinario de vendaje algodonoso en nuestro servicio, lo que limita la movilidad.

La estancia en recuperación es baja (de tan sólo 1.9 horas como promedio en el grupo A), sobre todo al comparar las

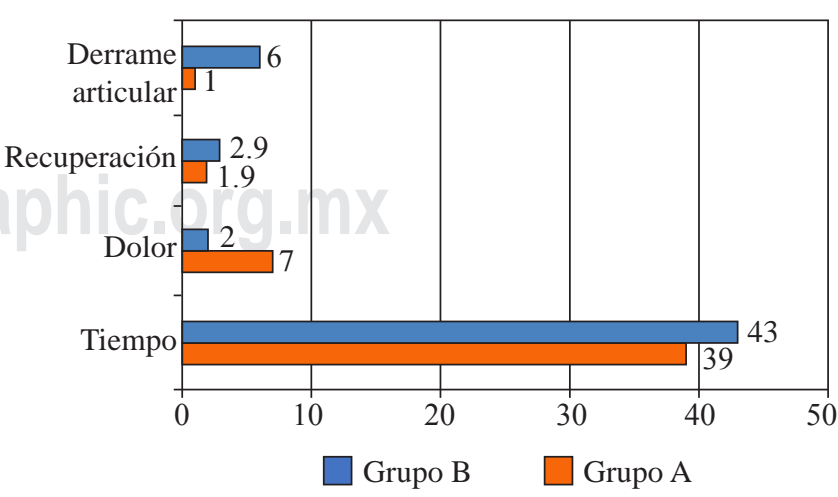

Figura 1: Presencia de derrame articular (número de pacientes), estancia promedio en recuperación (horas), presencia de dolor transquirúrgico (número de pacientes) y tiempo quirúrgico (minutos). 
Tabla 1: Resumen de las características de los pacientes entre grupos y resumen de los resultados con significancia: sexo, edad, tiempo quirúrgico, dolor intra- o transquirúrgico, tiempo en horas en recuperación, dolor en el postquirúrgico a las $\mathbf{2 4}$ horas, presencia de complicaciones postquirúrgicas, necesidad de hospitalización y grados de flexión a las $\mathbf{2 4}$ horas.

\begin{tabular}{|c|c|c|c|}
\hline Características de los pacientes & Espinal & Intraarticular & \\
\hline Sexo & $\begin{array}{l}\text { Femenino }=18 \\
\text { Masculino }=20\end{array}$ & $\begin{array}{c}\text { Femenino }=9 \\
\text { Masculino }=29\end{array}$ & \\
\hline Edad & 40 (rango 19-64) & 35 (rango 18-65) & \\
\hline Tiempo quirúrgico & 45.28 minutos (rango $25-120$ ) & 39.3 minutos (rango $25-55$ ) & $\mathrm{p}=0.03$ \\
\hline Dolor intraquirúrgico & 1 (EVA 4$)$ & 7 (EVA 4) & $\mathrm{p}=0.15$ \\
\hline Tiempo en recuperación & 3 horas (rango 0-5) & 1.9 horas (rango $1-4$ ) & $\mathrm{p}=0.0004$ \\
\hline Dolor postquirúrgico & EVA 1.5 (rango 0-4) & EVA 1.3 (rango 1-6) & $\mathrm{p}=0.09$ \\
\hline Complicaciones quirúrgicas & Derrame (6) & Derrame (1) & $\chi^{2}=0.2$ \\
\hline Hospitalización & 4 pacientes & 1 paciente & $\chi^{2}=0.5$ \\
\hline Flexión & $90(50-120)$ & $86(30-110)$ & $\mathrm{p}=0.12$ \\
\hline
\end{tabular}

tres horas promedio con el grupo B. Ésta no llega a ser tan baja como la reportada por Mondino, ${ }^{5}$ aunque la consideramos bastante buena. El dolor postquirúrgico que requiriera hospitalización se presentó en tres ocasiones en el grupo B $(10 \%)$ y sólo una (3\%) en el grupo A, lo cual consideramos adecuado. No hay más estudios que consideren la hospitalización para el control de dolor postquirúrgico, simplemente el uso de analgésicos o la mayor estancia de hospitalización en recuperación; Mondino refiere un promedio de estancia en «hospital de día» de 7.34 horas, con un rango de cuatro a 11 horas.

La presencia de incidentes postquirúrgicos, como derrame, la encontramos en pacientes que requirieron sinovectomía o microfractura y más comúnmente en pacientes con uso de bloqueo espinal (en seis pacientes [15\%]) que en la anestesia intraarticular (en un paciente [3\%]). No obstante, no podemos considerarla como un factor protector, debido a que la prueba $\chi^{2}$ es negativa. Mondino, ${ }^{5}$ Martínez Trejo ${ }^{8} 0$ Díaz Osuna ${ }^{10}$ no refieren la presencia de este incidente, sólo cefalea postpunción, la cual no encontramos nosotros.

El rango de flexión fue de 90 grados en promedio en el grupo A y de 86 grados en el grupo B, aunque su diferencia no fue significativa. En la bibliografía consultada, nadie realizó esta medición. Ningún paciente presentó efectos adversos a los medicamentos. Todos los pacientes podían elevar la extremidad operada y ningún paciente requirió anestesia general.

El uso de anestesia intraarticular es seguro y efectivo. El uso de fentanil, bupivacaína y lidocaína con epinefrina, aunque ya previamente estudiados, no se había comparado directamente con el procedimiento habitual en nuestro servicio (bloqueo espinal). El uso de anestesia intraarticular se asoció con un menor tiempo quirúrgico y un tiempo de latencia equivalente o casi equivalente al de la preparación del campo quirúrgico. Asimismo, se asoció con un menor dolor postquirúrgico y con una menor estancia en el servicio de recuperación. Sin embargo, tiene la desventaja de poder llegar a necesitarse dosis anestésica de rescate y el dolor transquirúrgico, sin que se llegue a necesitar aneste- sia general en éste u otro estudio similar., ${ }^{5,9}$ Aconsejamos su uso en aquellos pacientes que requieran una artroscopía diagnóstico-terapéutica ${ }^{5,8} \sin$ microfracturas, liberación del retináculo lateral y sinovectomías, ya que podrían requerir un mayor tiempo intrahospitalario de observación.

Bibliografía

1. Johnson D, Amendola N, Barber F. Operative arthroscopy. Philadelphia: Wolters Kluwer; 2015.

2. Shaukat Y, Malik E, El-Khateeb H, Koeweiden E. The role of local anaesthesia in knee arthroscopy. J Orthop. 2013; 10(4): 193-5. Disponible en: http://dx.doi.org/10.1016/j.jor.2013.06.003.

3. Zhou Y, Yang T Bao, Wei J, Zeng C, Li H, Yang T, et al. Single-dose intra-articular ropivacaine after arthroscopic knee surgery decreases post-operative pain without increasing side effects: a systematic review and meta-analysis. Knee Surgery, Sport Traumatol Arthrosc. 2016; 24(5): 1651-9.

4. Yang Y, Zeng C, Wei J, Li H, Yang T, Deng Z, et al. Single-dose intraarticular bupivacaine plus morphine versus bupivacaine alone after arthroscopic knee surgery: a meta-analysis of randomized controlled trials. Knee Surg Sports Traumatol Arthrosc. 2017; 25(3): 966-79.

5. Mondino JA. Artroscopia de rodilla con anestesia local. Artroscopia. 2006; 13(2): 102-10

6. De Santiago J, Santos-Yglesias J, Giron J, Jimenez A, Errando CL. Low-dose, low-concentration levobupivacaine plus fentanyl selective spinal anesthesia for knee arthroscopy: a dose finding study. Anesth Analg. 2011; 112(2): 477-80.

7. Bech NH, Hulst AH, Spuijbroek JA, Leuken LLA Van, Haverkamp D. Perioperative pain management in hip arthroscopy; what options are there? J Hip Preserv Surg. 2016; 3(3): 181-9.

8. Trejo MR, Valdés LM, Roldán FT. Artroscopía de la rodilla bajo anestesia local. Rev Mex Ortop Traum. 2000; 14(2): 191-5.

9. Rodríguez VA, Anguiano GM. Anestesia intra-articular en la artroscopía de la rodilla. Estudio experimental comparativo doble ciego. Rev Mex Ortop Traum. 2000; 14(192): 196-9.

10. Díaz-Osuna V, Vilchez-Cavazos F, Palacios-Ríos D, Ibarra-Malacara EA, Espinosa-Galindo AM. Local anesthesia and sedation vs. spinal anesthesia for knee arthroscopy. Med Univ. 2016; 18(72): 153-7. Disponible en: http://www.sciencedirect.com/science/article/pii/ S1665579616300850.

Financiamiento: Se usaron recursos provistos por la misma institución. 